\title{
ASPECTOS BIOLÓGICOS DE Phthia picta (DRURY, 1770) (HEMIPTERA: COREIDAE) EM TOMATEIRO SOB CONDIÇÕES CONTROLADAS
}

\author{
BIOLOGICAL ASPECTS OF Phthia picta (DRURY, 1770) \\ (HEMIPTERA: COREIDAE) ON TOMATOES UNDER CONTROLLED CONDITIONS
}

\author{
Ricardo Adaime da Silva ${ }^{1}$ Gervásio Silva Carvalho $^{2}$
}

RESUMO

Phthia picta (Drury, 1770) é considerado importante praga da cultura do tomateiro-estaqueado, em função dos danos que pode causar aos frutos. Devido à ausência de informações sobre a biologia desse coreídeo sob condições controladas, realizou-se este trabalho, objetivando o seu estudo em uma cultivar comercial de tomateiro (Lycopersicon esculentum Mill). O trabalho foi conduzido no Laboratório de Entomologia do Departamento de Fitossanidade da Faculdade de Agronomia da UFRGS, sob condições controladas $\left(26 \pm 1^{\circ} \mathrm{C} ; 70 \pm 10 \%\right.$ UR e fotofase de 14h), utilizando como alimento foliolos e frutos de tomateiro, cultivar Carmen. Os estádios duraram 3,26, 6,91, $7,91,7,12$ e 11,25 dias, respectivamente ao $1,2^{\circ}, 3^{\circ}, 4^{\circ}$ e $5^{\circ}$. A duração da fase ninfal foi de 34,39 dias. A mortalidade foi maior no $3^{\circ}, 4^{\circ}$ e $5^{\circ}$ estádios. A mortalidade na fase ninfal foi de 56,0\%. O número de ovos/fêmea, oviposições/fêmea e ovos/oviposição foi $60,43,6,14$ e 9,84, respectivamente. O período de incubação dos ovos foi de 10,90 dias e a percentagem de eclosão foi de 65,87\%.

Palavras-chave: Insecta, percevejo, Lycopersicon esculentum, laboratório.

\section{SUMMARY}

Phthia picta (Drury, 1770) is an important pest of the tomato culture. There are not studies on this bug under controlled conditions. So, this report was developed this work aiming at the study of this insect biology in a comercial cultivar of tomato (Lycopersicon esculentum Mill). The study was carried out in the Laboratório de Entomologia, Departamento de Fitossanidade, Faculdade de Agronomia, UFRGS, under controlled conditions $\left(26 \pm 1^{\circ} \mathrm{C} ; 70 \pm 10 \% \mathrm{RH}\right.$ and photophase of 14h), using as food leaves and fruits of the Carmen cultivar tomato. The 1st, 2nd, 3rd, 4th and 5th stages lasted 3.26, 6.91, 7.91, 7.12 and 11.25 days, respectively. The duration of the nymphal phase was 34.39 days. The mortality rate was higher in the $3 \mathrm{rd}$, 4th and 5th stages. In the nymphal phase the mortality was 56.0\%. The number of eggs/female, oviposition/female and eggs/oviposition was 60.43, 6.14 and 9.84, respectively. The egg incubation period was 10.90 days and the hatching percentage was $65.87 \%$.

Key words: Insecta, bug, Lycopersicon esculentum, laboratory.

\section{INTRODUÇÃO}

A cultura do tomateiro (Lycopersicon esculentum Mill) possui grande importância sócioeconômica para o Brasil, sendo que grande parte da produção de frutos para consumo "in natura" é proveniente de pequenas propriedades, nas quais a mão-de-obra é predominantemente familiar. Entre as pragas da cultura está Phthia picta (Drury, 1770), um dos coreídeos mais freqüentemente encontrados em todo o Brasil (LIMA, 1940), vulgarmente conhecido como "percevejo-do-tomate". A ocorrência de $\boldsymbol{P}$. picta inclui Califórnia, Texas (TORRE BUENO, 1941), Flórida, Antilhas, México, América do Sul (THOMAS, 1915) e Caribe (KING $\&$ SAUNDERS, 1984). Possui uma ampla gama de plantas hospedeiras, principalmente solanáceas e cucurbitáceas, preferindo o tomateiro (SERANTES, 1973), no qual se alimenta tanto nas folhas quanto

\footnotetext{
${ }^{1}$ Engenheiro Agrônomo, Mestre em Fitotecnia, Doutorando em Agronomia (Entomologia Agrícola), Faculdade de Ciências Agrárias e Veterinárias, Universidade Estadual Paulista, Jaboticabal, SP. E-mail: rica@fcav.unesp.br.

${ }^{2}$ Biólogo, Doutor, Professor Adjunto, Faculdade de Agronomia, Departamento de Fitossanidade, Universidade Federal do Rio Grande do Sul. Av. Bento Gonçalves, 7712, CP 776, 90012-970, Porto Alegre, RS, Brasil. E-mail: gerve@vortex.ufrgs.br. Autor para correspondência.
} 
nos frutos. Nestes, ninfas e adultos efetuam punções e sugam seu conteúdo (MARICONI, 1963), deixando-os suscetíveis ao ataque de outros insetos, além de microrganismos que apressam a sua deterioração (MONTE, 1932 e 1939). No local da penetração dos estiletes, ocorre uma necrose característica (VERNALHA et al., 1968). Devido aos danos que pode causar é referido como o coreídeo mais importante (CARRERA, 1973). Não existem estimativas numéricas dos danos causados pelo percevejo-do-tomate, no entanto, em termos qualitativos são significativos, sobretudo quando causados por adultos em frutos de mesa, para os quais a qualidade é fundamental. Diversos pesquisadores relataram que esse percevejo pode ser vetor de protozoários flagelados, mas esses nem sempre causam efeitos patológicos nas plantas (JANKEVICIUS et al., 1989; BRAZIL et al., 1990; FIORINI et al., 1993). No entanto, são necessários mais estudos a esse respeito para elucidar tal questão.

Alguns aspectos biológicos e morfológicos de $\boldsymbol{P}$. picta sob condições não controladas foram estudados por SERANTES (1973), em Buenos Aires, Argentina. Os insetos foram alimentados com diversas cucurbitáceas e solanáceas, em especial o tomateiro. AMARAL FILHO (1980; 1981a; 1981b) estudou aspectos biológicos de adultos acasalados e não acasalados desse coreídeo, o efeito de dietas naturais na sua biologia e alguns aspectos comportamentais.

Este trabalho teve o objetivo de estudar aspectos biológicos de $\boldsymbol{P}$. picta sob condições controladas, alimentado com folíolos e frutos de tomateiro da cultivar comercial Carmen.

\section{MATERIAL E MÉTODOS}

O trabalho foi realizado no Laboratório de Entomologia e na Área Experimental do Departamento de Fitossanidade da Faculdade de Agronomia da Universidade Federal do Rio Grande do Sul, Porto Alegre (30 $01^{\prime} \mathrm{S}$ e $51^{\circ} 13^{\prime} \mathrm{W}$ ), Rio Grande do Sul.

Durante a safra de 1998/99, foi conduzida uma plantação de tomate, cultivar híbrida Carmen (tipo longa-vida), onde foram coletados ovos, ninfas e adultos de $\boldsymbol{P}$. picta e iniciou-se a sua criação massal. Ovos, ninfas e adultos foram mantidos em estufa incubadora, sob temperatura de $26 \pm 1^{\circ} \mathrm{C}$, umidade relativa do ar de $70 \pm 10 \%$ e fotofase de 14 horas.

A criação das ninfas foi realizada agrupadamente, visando a evitar o estresse por manipulação excessiva, tentando reproduzir em laboratório o que ocorre na natureza, isto é, comportamento gre- gário nos estádios iniciais. Os grupos eram formados por ninfas de mesma idade, provenientes de um mesmo grupo de ovos, permanecendo agrupadas enquanto não sofressem nova muda. Quando sofriam muda, as ninfas eram removidas e agrupadas em outro frasco. Alguns casos ocorreram de ninfas de $4^{\circ}$ e $5^{\circ}$ estádios serem criadas isoladamente. Isso ocorreu, por exemplo, quando o grupo era composto de poucas ninfas de $4^{\circ}$ estádio e apenas uma delas sofreu ecdise em determinado dia. Foram utilizados frascos de plástico transparente, de dois tamanhos, cobertos com malha sintética denominada "tunyl", presa por atilhos de borracha. Para grupos de até 15 ninfas, foram utilizados frascos de $6,5 \mathrm{~cm}$ de base $\mathrm{x}$ $8,5 \mathrm{~cm}$ de abertura $x 7,5 \mathrm{~cm}$ de altura. Para grupos de mais de 15 ninfas, foram utilizados frascos de $8,5 \mathrm{~cm}$ $\mathrm{x} 11,5 \mathrm{~cm} \times 8 \mathrm{~cm}$. À medida que eclodiam as ninfas, eram oferecidos, no interior de cada frasco, um fruto verde e um folíolo, cujo pecíolo estava imerso em água contida em pequeno frasco de vidro com tampa vazada. Considerando-se o longo período ninfal, os frutos amadureciam progressivamente, sendo, muitas vezes, necessária a sua substituição. Quando isso ocorria, procurava-se dispor aos insetos frutos no mesmo estágio de maturação, simulando uma sincronia entre o desenvolvimento do fruto e do inseto. As ninfas foram observadas diariamente, registrando-se as mortas, removendo-se as exúvias e transferindo-se as que sofriam muda para outro frasco. Com o acompanhamento diário de todos os frascos, foi possível obter dados de mortalidade e de duração de cada estádio, para ninfas de segunda e terceira gerações de laboratório. Quando atingiam a fase adulta, os insetos eram separados por sexo e contados a fim de se calcular a proporção de machos por fêmea e a razão sexual.

Os parâmetros reprodutivos e a longevidade foram observados em adultos de segunda geração de laboratório. Para tanto, foram formados 12 casais, no máximo três dias após a emergência dos insetos, acondicionados em frascos de $8,5 \mathrm{~cm} \mathrm{x}$ $11,5 \mathrm{~cm} \times 8 \mathrm{~cm}$, cobertos com "tunyl". No interior de cada frasco, foram oferecidos um folíolo e um fruto, de forma análoga à descrita para ninfas. Diariamente os frascos foram vistoriados, registrando-se o número de ovos presentes e o local de postura. Foram avaliados os períodos de pré-oviposição, oviposição, pós-oviposição, além do número de ovos postos por fêmea, número de posturas por fêmea, número de ovos por postura e a longevidade dos adultos.

Os ovos oriundos de insetos de segunda e terceira gerações de laboratório foram mantidos em frascos de plástico transparente de $6,5 \mathrm{~cm} \times 8,5 \mathrm{~cm} \times$ $7,5 \mathrm{~cm}$ e acompanhados diariamente, a fim de registrar o período de incubação e a percentagem de eclosão de ninfas. 
Sempre que necessário, realizou-se a limpeza dos frascos, com o auxílio de pinça e algodão embebido em álcool etílico $70 \%$, a fim de manter boa higiene na criação.

Os dados de mortalidade em cada estádio e na fase ninfal foram analisados pelo Teste $\chi^{2}$ a dados de enumeração a $5 \%$. Os dados de duração de cada estádio e da fase ninfal foram submetidos à Análise de Variância e, quando necessário, esta foi complementada com o Teste de Duncan a 5\%. Cada inseto foi considerado uma repetição em Delineamento Completamente Casualizado. Foi observada a longevidade tanto de casais cujas fêmeas produziram ovos quanto dos casais cujas fêmeas não produziram, sendo submetidos à Análise de Variância em esquema fatorial (fator A: produção de ovos; fator B: sexo)

\section{RESULTADOS E DISCUSSÃO}

Todos os indivíduos que atingiram a fase adulta apresentaram cinco estádios ninfais. A duração da fase ninfal foi de 34,39 dias. Os dados obtidos distanciam-se dos apresentados por SERANTES (1973), que refere a duração da fase ninfal de $\boldsymbol{P}$. picta como sendo de 25 dias (temperaturas variando entre 15 e $25^{\circ} \mathrm{C}$ ); e dos obtidos por AMARAL FILHO (1981a), 54,87 dias para ninfas criadas agrupadas (temperatura mínima de $24,60^{\circ} \mathrm{C}$ e máxima de $29,74^{\circ} \mathrm{C}$ ) e 75,66 dias para ninfas criadas isoladas (temperatura mínima de $23,03^{\circ} \mathrm{C}$ e máxima de $\left.30,38^{\circ} \mathrm{C}\right)$. Essa discrepância, provavelmente, esteja relacionada às diferentes condições de temperatura em que os experimentos foram realizados, às técnicas de criação e ao tipo de alimentação.

$\mathrm{O}$ estádio mais curto foi o $1^{\mathrm{o}}$ e o mais longo foi o $5^{\mathrm{o}}$ (Tabela 1$)$. Isso corrobora a afirmação de PANIZZI (1991), de que os hemípteros requerem um tempo maior para completar o $5^{-}$estádio, em comparação com os estádios anteriores. O $3^{\circ}$ estádio ninfal teve maior duração que o $2^{\circ}$ e o $4^{\circ}$, estes não diferindo entre si. AMARAL FILHO (1981a) obteve 4,64 dias de duração do $1^{\circ}$ estádio ninfal de $\boldsymbol{P}$. picta, criando ninfas agrupadas, sendo alimentadas com pedaços de fruto de tomateiro colocados sobre a malha sintética que cobria os frascos de criação. Ao autor não foi possível avaliar a duração dos demais estádios. Ao criar ninfas isoladas, com a mesma alimentação, obteve duração dos cinco estádios ninfais de 3,88,11,80,18,48, 19,30, 26,33 dias, respectivamente. Os dados provenientes de ninfas criadas isoladas diferem bastante dos dados obtidos no presente trabalho, devendo-se considerar que o inseto é gregário nos estádios iniciais, de modo que a criação das ninfas de forma isolada compromete o
Tabela 1 - Média ( \pm EP) e intervalo de confiança (dias) da duração dos estádios e da fase ninfal, e percentual de mortalidade nos estádio e na fase ninfal de Phthia picta (Drury, 1770), alimentado com folíolos e frutos de tomateiro $\left(26 \pm 1^{\circ} \mathrm{C} ; 70 \pm 10 \%\right.$ UR $)$. Porto Alegre, RS. 1999.

\begin{tabular}{|c|c|c|c|}
\hline Estádio & $\begin{array}{c}\text { Duração média }^{1} \\
\pm \mathrm{EP}\end{array}$ & $\begin{array}{c}\text { Intervalo de } \\
\text { Confiança } 99 \%\end{array}$ & $\begin{array}{c}\text { Mortalidade }^{2} \\
\%\end{array}$ \\
\hline $1^{\circ}$ & $\begin{array}{c}3,26 \pm 0,04 \mathrm{~d} \\
(156)^{3}\end{array}$ & {$[3,17 ; 3,35]$} & $\begin{array}{c}0 \\
(156)\end{array}$ \\
\hline $2^{\circ}$ & $\begin{array}{c}6,91 \pm 0,12 \mathrm{c} \\
(147)\end{array}$ & {$[6,59 ; 7,23]$} & $\begin{array}{c}5,8 \text { B } \\
(156)\end{array}$ \\
\hline $3^{\circ}$ & $\begin{array}{c}7,91 \pm 0,34 b \\
(106)\end{array}$ & {$[6,43 ; 8,17]$} & $\begin{array}{l}18,5 \mathrm{~A} \\
(130)\end{array}$ \\
\hline $4^{\circ}$ & $\begin{array}{c}7,12 \pm 0,22 \mathrm{c} \\
(76)\end{array}$ & {$[6,57 ; 7,69]$} & $\begin{array}{l}26,9 \mathrm{~A} \\
(104)\end{array}$ \\
\hline $5^{\circ}$ & $11,25 \pm \underset{(59)}{ \pm 0,37 a}$ & {$[10,30 ; 12,19]$} & $\begin{array}{l}20,3 \mathrm{~A} \\
(74)\end{array}$ \\
\hline Fase ninfal & $\begin{array}{c}34,39 \pm 0,67 \\
(59)\end{array}$ & {$[32,66 ; 36,12]$} & $\begin{array}{l}56,0 \\
(134)\end{array}$ \\
\hline
\end{tabular}

${ }^{1}$ Médias seguidas de mesma letra minúscula na coluna não diferem significativamente entre si. Duncan 5\%.

${ }^{2}$ Médias seguidas de mesma letra maiúscula na coluna não diferem significativamente entre si. Teste $\chi^{2}$ a $5 \%$.

${ }^{3}$ Número de ninfas entre parênteses.

seu desenvolvimento, podendo resultar em estádios mais longos.

Nas condições de Buenos Aires, Argentina, os cinco estádios ninfais de $\boldsymbol{P}$. picta têm duração de 3-4, 4, 4, 6 e 7 dias, respectivamente (SERANTES, 1973). A autora, entretanto, não cita o número de insetos avaliados em cada estádio e a variabilidade dos dados. Assim, somente a duração registrada para o $1^{\circ}$ estádio se assemelha à obtida no presente trabalho. A autora relata que diversos exemplares permaneceram por, aproximadamente, 60 dias no $5^{\circ}$ estádio, mas que a duração média foi de 7 dias. Esses dados diferem dos obtidos no presente trabalho, no qual a duração foi de 11,25 dias. Entre os insetos que iniciaram o $5^{-}$estádio mas morreram antes de atingir a fase adulta, a média de vida foi de 19,67 dias.

No $1^{\underline{0}}$ estádio ninfal, não houve mortalidade (Tabela 1). As mortalidades ocorridas no $3^{\circ}, 4^{\circ}$ e $5^{\circ}$ estádios não diferiram entre si, sendo superiores às ocorridas no $2^{\circ}$ estádio. Considerando-se ninfas que tiveram chances de atingir a fase adulta (ou seja, não foram descartadas antes de morrerem ou passarem para o estádio seguinte), a mortalidade na fase ninfal foi de 56,0\%. Estudando alguns aspectos biológicos de $\boldsymbol{P}$. picta, AMARAL FILHO (1981a) obteve $2 \%$ de mortalidade no $1^{\circ}$ estádio em ninfas criadas agrupadas, não fornecendo os valores para os demais estádios. Em ninfas criadas isoladamente as mortalidades foram de $6,46 \%, 58,25 \%, 80,57 \%$, 
$51,85 \%$ e $76,92 \%$, para os cinco estádios, respectivamente. A alta mortalidade é comum em coreídeos (PANIZZI, 1989), devendo-se considerar que parte dessa mortalidade pode ser atribuída a fatores intrínsecos da espécie (AMARAL FILHO, 1981a).

O gregarismo é uma adaptação para a alimentação, tendo um efeito positivo na sobrevivência das ninfas (PANIZZI, 1991). Durante a execução do presente trabalho, observou-se que as ninfas permaneceram agrupadas nos folíolos principalmente no $1^{\circ}$ estádio, no $2^{-}$, dispersaram-se e procuraram o fruto para se alimentar. Comparando-se as mortalidades registradas no presente trabalho com as obtidas por AMARAL FILHO (1981a) com insetos criados isoladamente, pode-se inferir que a técnica de criação de insetos de mesmo estádio agrupados é mais adequada, por propiciar a fase ninfal mais curta e menor percentual de mortalidade.

A proporção de machos e fêmeas foi de 1 : 0,97 , o que resulta numa razão sexual igual a 0,49 , ou seja o número de fêmeas na população, de $49,1 \%$. Os dados distanciam-se dos obtidos por AMARAL FILHO (1978), que registrou proporção sexual de 1 macho: 1,55 fêmeas.

De 12 fêmeas acasaladas, 7 produziram ovos $(58,33 \%)$. Foi observada a longevidade tanto de casais cujas fêmeas produziram ovos quanto dos casais cujas fêmeas não produziram, submetendo-se os dados à Análise de Variância (Teste F) em esquema fatorial, estando as médias presentes na tabela 2. A análise indicou que entre os casais cujas fêmeas produziram ovos, a longevidade de fêmeas e machos foi significativamente diferente entre si ( $\mathrm{F}=$ 6,3709). Entre os casais cujas fêmeas não produziram ovos, a longevidade de fêmeas e machos não foi diferente entre si $(F=0,2952)$. Fêmeas que produziram ovos viveram significativamente mais que as que não produziram $(\mathrm{F}=6,3236)$. $\mathrm{O}$ fato das fêmeas que acompanhavam os machos produzirem ou não ovos, não influenciou a longevidade deles $(\mathrm{F}=0,2110)$. SERANTES (1973) refere que adultos de $\boldsymbol{P}$. picta vivem 33-35 dias, havendo registrado casos de até 70 dias. Entre indivíduos acasalados, AMARAL FILHO (1980) registrou longevidade de 59,0 dias para fêmeas e de 55,46 dias para machos, não havendo diferença significativa entre os sexos. Entre indivíduos não acasalados, registrou 36,82 dias para fêmeas e 49,71 dias para machos, não havendo diferença significativa entre os sexos. $\mathrm{O}$ autor registrou diferença significativa quanto à longevidade entre insetos acasalados e não acasalados.

Dentre as posturas efetuadas por insetos de segunda geração de laboratório, a maioria dos ovos foi depositada sobre os folíolos de tomateiro (54,76\% dos ovos). Houve, também, percentual representativo de ovos depositados nos frascos de criação, malha sintética de cobertura dos frascos e no vidro que sustentava os folíolos. Os resultados são semelhantes aos observados por AMARAL FILHO (1981b), de que os ovos de $\boldsymbol{P}$. picta podem ser depositados em qualquer substrato. Porém, os dados obtidos denotam uma tendência para os folíolos de tomateiro.

De 420 ovos avaliados, 39,72\% foram depositados em fileira única, $19,15 \%$ em duas fileiras, $20,80 \%$ em três fileiras e $20,33 \%$ em quatro fileiras. Essas fileiras podem ser paralelas ou não, havendo forte aderência dos ovos entre si e em relação ao substrato. Em 43 posturas observadas, o número médio de ovos foi $9,84 \pm 0,41$. Posturas de apenas um ovo corresponderam a $6,98 \%$ do total e o maior número de ovos registrados em uma postura foi 34 . SERANTES (1973) registrou 10-20 ovos por postura, tendo encontrado uma postura com 82 ovos em fileira única. Avaliando 95 posturas, AMARAL FILHO (1980) registrou uma média de 18,10 ovos por postura. Dessa forma, as médias obtidas no presente trabalho são inferiores às obtidas pelos autores supracitados.

O número de ovos e de

\begin{tabular}{lcc}
\hline Categoria & Fêmeas & Machos \\
\hline Casais cujas fêmeas produziram ovos ${ }^{1,2}$ & $120,83 \pm 18,86 \mathrm{aA}$ & $73,33 \pm 10,15 \mathrm{~b} \mathrm{~A}$ \\
& $(6)^{3}$ & $(6)$ \\
Casais cujas fêmeas não produziram ovos & $71,20 \pm 13,98 \mathrm{a}$ B & $82,40 \pm 8,44 \mathrm{aA}$ \\
& $(5)$ & $(5)$ \\
\hline
\end{tabular}

${ }^{1}$ Médias seguidas de mesma letra minúscula na linha não diferem significativamente. Teste F a $5 \%$.

${ }^{2}$ Médias seguidas de mesma letra maiúscula na coluna não diferem significativamente. Teste F a $5 \%$.

${ }^{3}$ Número de insetos entre parênteses. posturas por fêmea foi de 60,43 e de 6,14 , respectivamente (Tabela $3)$. Esses resultados aproximam-se dos registrados por SERANTES (1973) e AMARAL FILHO (1980), $35-45$ e 66,15 ovos por fêmea, e 24 e 3,65 posturas por fêmea, respectivamente. Os períodos de pré-oviposição, oviposição e pós- 
Tabela 3 - Duração dos períodos de pré-oviposição, oviposição e pós-oviposição e intervalo entre oviposições ( $\pm \mathrm{EP}$ ), em dias, de Phthia picta (Drury, 1770), alimentado com folíolos e frutos de tomateiro $\left(26 \pm 1^{\circ} \mathrm{C} ; 70 \pm\right.$ 10\% UR). Porto Alegre, RS. 1999.

\begin{tabular}{|c|c|c|}
\hline Período & $\begin{array}{l}\text { Duração } \\
\text { (dias) }\end{array}$ & $\begin{array}{l}\text { Amplitude } \\
\text { (dias) }\end{array}$ \\
\hline Pré-oviposição & $62,83 \pm \frac{ \pm}{(6)}$ & $29-99$ \\
\hline Oviposição & $47,40 \pm \underset{(5)}{ \pm} 11,95$ & $18-90$ \\
\hline Pós-oviposição & $6,50 \pm \frac{ \pm}{(5)} 1,89$ & $2-13$ \\
\hline Intervalo entre oviposições & $9,37 \pm \underset{(6)}{ \pm} 1,48$ & $5,22-16,00$ \\
\hline
\end{tabular}

${ }^{1}$ Número de fêmeas entre parênteses.

oviposição e o intervalo entre oviposições foram de $62,83,47,40,6,50$ e 9,37 dias, respectivamente. AMARAL FILHO (1980) registrou para esses períodos os seguintes valores: $24,69,24,61,10,88$ e 9,04 dias, respectivamente. Os valores para os períodos de pré-oviposição e de oviposição obtidos no presente trabalho são maiores que os registrados pelo pesquisador supracitado, e os valores para o período de pós-oviposição e intervalo entre oviposições são bastante semelhantes.

O período médio de incubação dos ovos foi de 10,90 $\pm 0,06$ dias $(n=166)$, semelhante aos obtidos por SERANTES (1973) e AMARAL FILHO (1981a), 6-10 dias e 10,39 dias, respectivamente. O percentual de eclosão foi de $65,87 \%(n=252)$, sendo inferior aos valores obtidos por SERANTES (1973) e AMARAL FILHO (1981a), 98\% e 86,85\%, respectivamente. Houve eclosão de ninfas do $10^{\circ}$ ao $12^{-}$dia de incubação, havendo o pico no $11^{\circ}$ dia, representando $46,99 \%$ dos ovos.

Em função dos danos que $\boldsymbol{P}$. picta pode causar a frutos de tomateiro destinados ao consumo in natura, sobretudo quando se pensa em cultivo sem o uso de agrotóxicos, e da ausência de informações disponíveis na literatura acerca de sua bioecologia em condições de campo, sugere-se que sejam realizados estudos com essa praga em cultivos comerciais nas regiões produtoras.

\section{CONCLUSÕES}

Nas condições em que o ensaio de biologia de $\boldsymbol{P}$. picta foi conduzido e com base nos resultados obtidos, são possíveis as seguintes conclusões: o $1^{\circ}$ estádio é o mais curto e o $5^{\circ}$ o mais longo; os maiores percentuais de mortalidade ocorrem no $3^{\circ}$, $4^{\circ}$ e $5^{\circ}$ estádios ninfais, não sendo distintos entre si; fêmeas de segunda geração de laboratório não apresentam alto potencial reprodutivo; entre as fêmeas acasaladas, as que produzem ovos vivem significativamente mais que as que não produzem.

\section{REFERÊNCIAS BIBLIOGRÁFICAS}

AMARAL FILHO, B.F. Contribuição ao conhecimento da biologia de Phthia picta (Drury, 1770) (Hemiptera, Coreidae) sob condições de laboratório. São Paulo, 1978. 89p. Dissertação (Mestrado em Zoologia) - Instituto de Biociências, Universidade de São Paulo, 1978.

AMARAL FILHO, B.F. Observações biológicas em adultos acasalados e não acasalados de um coreídeo praga - Phthia picta (Drury, 1770). Anais da Sociedade Entomológica do Brasil, Jaboticabal, v.9, n.1, p.67-74, 1980.

AMARAL FILHO, B.F. Efeito de dietas naturais e de fatores ambientais na biologia de Phthia picta (Drury, 1770) sob condições de laboratório (Hemiptera, Coreidae). Revista Brasileira de Biologia, Rio de Janeiro, v.41, n.4, p.845-853, 1981a.

AMARAL FILHO, B.F. Aspectos comportamentais de Phthia picta (Drury, 1770) em condições de laboratório (Hemiptera, Coreidae). Revista Brasileira de Biologia, Rio de Janeiro, v.41, n.2, p.441-446, 1981b.

BRAZIL, R.P., FIORINI, J.E., FARIA-E-SILVA, P.M. Phytomonas sp., a trypanosomatid parasite of tomato, isolated from salivary glands of Phthia picta (Hemiptera: Coreidae) in southeast Brazil. Memórias do Instituto Oswaldo Cruz, Rio de Janeiro, v.85, n.2, p.239-240, 1990.

CARRERA, M. Entomologia para você. 4 ed. São Paulo : Edart, 1973. 185p.

FIORINI, J.E., DE-FARIA-E-SILVA, P.M., BRAZIL, R.P., $\boldsymbol{e t}$ al . Axenic cultivation of a pathogenic Phytomonas species isolated from tomato fruit, and from its phytophagic insect vetor, Phthia picta (Hemiptera: Coreidae). Cytobios, Cambridge, v.75, n.302-303, p.163-170, 1993.

JANKEVICIUS, J.V., JANKEVICIUS, S.I., CAMPANER, M., $\boldsymbol{e}$ t al. Life cycle and culturing Phytomonas serpens (Gibbs), a trypanosomatid parasite of tomatoes. Journal of Protozoology, Lawrence, v.36, n.3, p.265-271, 1989.

KING, A.B.S., SAUNDERS, J.L. The invertebrate pests of annuals food crops in Central America: a guide to their recognition and control. London : Overseas Development Administration, 1984. 166p.

LIMA, A.M. da C. Insetos do Brasil. Rio de Janeiro : Escola Nacional de Agronomia, 1940. T.2, 351p.

MARICONI, F.A.M. Inseticidas e seu emprego no combate às pragas. São Paulo: Ceres, 1963. 607p.

MONTE, O. Um percevejo sugador de tomate - Phthia picta Drury. Chácaras e Quintaes, São Paulo, v.45, n.2, p.222224, 1932.

Ciência Rural, v. 31, n. 3, 2001. 
MONTE, O. Hemípteros fitófagos. Campo, Rio de Janeiro, v.10, n.111, p.69-72, 1939 .

PANIZZI. A.R. Desempenho de ninfas e adultos de Leptoglossus zonatus (Dallas, 1852) (Hemiptera: Coreidae) em diferentes alimentos. Anais da Sociedade Entomológica do Brasil, Porto Alegre, v.18, n.2, p.375-389, 1989.

PANIZZI, A.R. Ecologia nutricional de insetos sugadores de sementes. In: PANIZZI, A.R., PARRA, J.R.P. Ecologia nutricional de insetos e suas implicações no manejo de pragas. São Paulo : Manole, 1991. p.253-287.

SERANTES, H.E. Biologia de Phthia picta (Drury) (Hemiptera, Coreidae). Fitotecnia Latinoamericana, Buenos Aires, v.9, n.1, p.3-9, 1973
THOMAS, J.R.T.B. Nota sobre hemípteros del Chaco de Santiago del Estero (República Argentina). Anales del Museo Nacional de Historia Natural de Buenos Aires, Buenos Aires, v.26, p.157-162, 1915.

TORRE-BUENO, J.R. de la. A synopsis of the HemipteraHeteroptera of America north of Mexico. Part II. Entomologica Americana, Lancaster, v.21, n.2, p.41-122, 1941.

VERNALHA, M.M., da ROCHA, M.A.L., GABARDO, J.C., $\boldsymbol{e t}$ al. Principais pragas das plantas cultivadas no Estado do Paraná. Curitiba : Escola de Agronomia e Veterinária da UFPR, 1968. 222p.

Ciência Rural, v. 31, n. 3, 2001. 